\section{Commentary: A step further to avoid allogenic transfusions in complex aortic surgery}

\author{
Carlos A. Mestres, MD, PhD, FETCS, ${ }^{\mathrm{a}, \mathrm{b}}$ and \\ Eduard Quintana, MD, PhD, FETCS ${ }^{\mathrm{C}}$
}

\begin{abstract}
Allogenic blood products make hemostatic control after aortic surgery feasible. The fight against blood loss and transfusion is aimed at decreasing associated morbidity and mortality. ${ }^{1}$ Aortic operations requiring hypothermic circulatory arrest (HCA) are examples of traumatic operations for which any blood-sparing strategy is beneficial. Autologous whole blood (AWB) collection is one of these strategies attempting to decrease allogeneic blood transfusion, and its efficacy remains under investigation. ${ }^{2,3}$
\end{abstract}

In this issue of the Journal, Geube and colleagues ${ }^{4}$ evaluate the effect of AWB on intraoperative and postoperative allogeneic blood transfusion in complex aortic surgery with HCA between 2014 and 2019. The authors matched 102 pairs to compare outcomes with AWB and without AWB, and concluded that pre-pump AWB collection may reduce the need for intraoperative transfusion of allogenic blood products. However, this immediate intraoperative benefit diminishes afterward, and the only difference in the need for fresh-frozen plasma is seen when considering the entire hospital stay. The indications for postoperative transfusion are not clearly described. The authors could not control transfusion records in the first

From the a Department of Cardiac Surgery, University Hospital Zürich, Zürich, Switzerland; ' Department of Cardiothoracic Surgery, University of the Free State, Bloemfontein, South Africa; and ${ }^{\mathrm{c}}$ Department of Cardiovascular Surgery, Hospital Clinic, University of Barcelona, Barcelona, Spain.

Disclosures: Dr Mestres has received speaker fees from the Edwards Clinical Events Committee (CEC) and Cytosorbents Corp. Dr Quintana reported no conflicts of interest.

The Journal policy requires editors and reviewers to disclose conflicts of interest and to decline handling or reviewing manuscripts for which they may have a conflict of interest. The editors and reviewers of this article have no conflicts of interest.

Received for publication Jan 22, 2021; revisions received Jan 22, 2021; accepted for publication Jan 25, 2021; available ahead of print Feb 2, 2021.

Address for reprints: Carlos A. Mestres, MD, PhD, FETCS, Department of Cardiac Surgery, University Hospital Zürich, Rämistrasse 100, CH-8091 Zürich, Switzerland (E-mail: Carlos.Mestres@usz.ch).

J Thorac Cardiovasc Surg 2022;164:1581-2

$0022-5223 / \$ 36.00$

Copyright $(2021$ by The American Association for Thoracic Surgery

https://doi.org/10.1016/j.jtcvs.2021.01.076

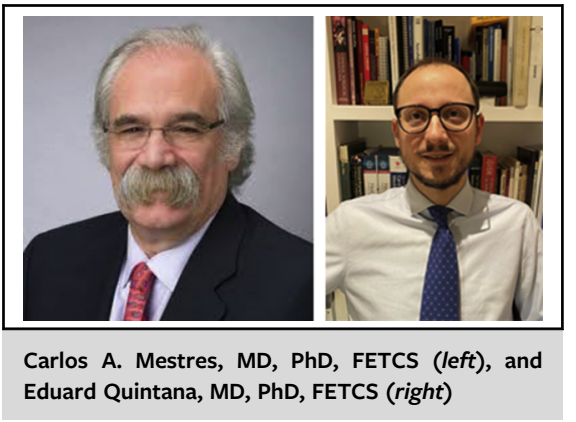

CENTRAL MESSAGE

Pre-pump autologous whole

blood collection is a strategy that may be useful in decreasing allogeneic blood transfusion in complex aortic surgery, but the full benefits need to be confirmed.

48 hours after surgery. There were no differences in hospital mortality, hospital length of stay, or postoperative complications between groups. Nonetheless, this study sheds some light on a segmental basis. In the operating room, autologous blood and derivative transfusions can initially be avoided.

Even at top aortic surgery practices like the authors', multiple blood product transfusions are the norm in such situations. We are confronted with this in daily practice, and every effort at improvement is much appreciated. ${ }^{5,6} \mathrm{We}$ share the authors' hypothesis that preventing exposure of reserved autologous blood to cardiopulmonary bypass (CPB) may preserve native hemostatic properties and decrease the need for allogenic transfusions.

One potential bias may have influenced the authors' results. Despite propensity matching, CPB and circulatory arrest times were significantly prolonged in the AWB group. Is this the result of assessing outcomes of different types of operations? Does this unexplained difference compromise the interpretation of data? Longer $\mathrm{CPB}$ and visceral ischemia times may translate into more coagulopathy. In addition, increased transfusion also may arise from higher technical complexity. If so, both would diminish the slight, albeit positive, benefits of AWB observed in this retrospective analysis. To further understand and judge the results, more in-depth scrutiny of the data on postoperative transfusion is needed. 
No holes in a blood vessel, no bleeding. Unfortunately, we make holes that need to be compensated for in such operations. The experienced surgeon is aware of the innumerable details influencing coagulopathy, blood loss, and need for transfusion, which are unlikely to be controlled in the setting of a retrospective analysis. Even though the reported impact of AWB appears to be only slightly favorable, its safety and plausibility support its adoption. Randomized trials are needed to assess the impact of various strategies following the lead of this experienced team.

The ability to perform complex aortic surgeries without the need for allogenic transfusion is the ultimate blood conservation goal toward which we strive. This work advances the journey toward transfusion-free aortic surgery.

\section{References}

1. Koch CG, Li L, Duncan AI, Mihaljevic T, Cosgrove DM, Loop FD, et al. Morbidity and mortality risk associated with red blood cell and blood-component transfusion in isolated coronary artery bypass grafting. Crit Care Med. 2006;34:1608-16.

2. Henderson RA, Mazzeffi MA, Strauss ER, Williams B, Wipfli C, Dawood M, et al. Impact of intraoperative high-volume autologous blood collection on allogeneic transfusion during and after cardiac surgery: a propensity score matched analysis. Transfusion. 2019;59:2023-9.

3. Rottman G, Ness PM. Acute normovolemic hemodilution is a legitimate alternative to allogeneic blood transfusion. Transfusion. 1998;38:477-80.

4. Geube M, Sale S, Bakdash S, Rajeswaran J, Roselli E, Blackstone E, et al. Prepump autologous blood collection is associated with reduced intraoperative transfusions in aortic surgery with circulatory arrest: a propensity score-matched analysis. J Thorac Cardiovasc Surg. 2022;164:1572-80.e5.

5. Spahn DR, Moch H, Hofmann A, Isbister JP. Patient blood management: the pragmatic solution for the problems with blood transfusions. Anesthesiology. 2008; 109:951-3.

6. Spahn DR, Muñoz M, Klein AA, Levy JH, Zacharowski K. Patient blood management: effectiveness and future potential. Anesthesiology. 2020;133:212-22.
See Article page 1572.

\section{Commentary: Autologous blood transfusion effects}

\author{
Hitoshi Hirose, MD, PhD, and Ashley Jaekel, CRNP
}

It was with great pleasure to review the article from Geube and colleagues ${ }^{1}$ from the Cleveland Clinic Foundation, entitled "Pre-Pump Autologous Blood Collection Is Associated With Reduced Intraoperative Transfusions in Aortic Surgery With Circulatory Arrest: A Propensity-Matched Analysis." The authors evaluated the effect of autologous whole blood (AWB) use in the elective, complex aortic surgery requiring hypothermic circulatory arrest. According to their protocol, AWB was collected shortly after induction and central-line placement and then transfused after protamine was administered to the patient. The decision to use AWB was decided on by the surgeon and anesthesiologist. This
Check for updates

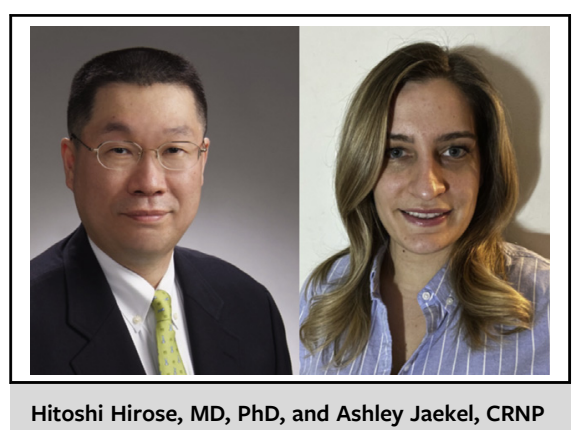

CENTRAL MESSAGE

Autologous whole-blood trans-

fusion can reduce intraoperative

allogenic transfusion even in

complex aortic surgery.
From the Department of Surgery, Thomas Jefferson University, Philadelphia, Pa. Disclosures: The authors reported no conflicts of interest.

The Journal policy requires editors and reviewers to disclose conflicts of interest and to decline handling or reviewing manuscripts for which they may have a conflict of interest. The editors and reviewers of this article have no conflicts of interest.

Received for publication Jan 21, 2021; revisions received Jan 21, 2021; accepted for publication Jan 22, 2021; available ahead of print Jan 30, 2021.

Address for reprints: Hitoshi Hirose, MD, PhD, Department of Surgery, Thomas Jefferson University, 1025 Walnut St, Ste 605, Philadelphia, PA 19107 (E-mail: Hitoshi.Hirose@jefferson.edu).

J Thorac Cardiovasc Surg 2022;164:1582-3

$0022-5223 / \$ 36.00$

Copyright (c) 2021 by The American Association for Thoracic Surgery

https://doi.org/10.1016/j.jtcvs.2021.01.085 retrospective study included 110 patients who received AWB over a 5-year period of time. Propensity matching was used to standardize the results, and authors were able to identify 95 well-matched pairs. The comparison between AWB versus non-AWB group was that the AWB group had a decreased number of transfusions including red blood cell (33\% vs $49 \%, P=.02)$, plasma (35\% vs $62 \%, P=.0002)$, platelets $(72 \%$ vs $82 \%, P=.09)$, and cryoprecipitate $(56 \%$ vs $63 \%, P=.3$ ) intraoperatively. ${ }^{1}$ Furthermore, despite 35 matched perioperative variables, the AWB group had longer 